\title{
HAMILTONIAN CIRCLE ACTIONS WITH ALMOST MINIMAL ISOLATED FIXED POINTS
}

\author{
HUI LI
}

\begin{abstract}
Let the circle act in a Hamiltonian fashion on a connected compact symplectic manifold $(M, \omega)$ of dimension $2 n$. Then the $S^{1}$ action has at least $n+1$ fixed points. In a previous paper, we study the case when the fixed point set consists of precisely $n+1$ isolated points. In this paper, we study the case when the fixed point set consists of exactly $n+2$ isolated points. We show that in this case $n$ must be even. We find equivalent conditions on the first Chern class of $M$ and a particular weight of the $S^{1}$-action. We also show that the particular weight can completely determine the integral cohomology ring and the total Chern class of $M$, and the sets of weights of the $S^{1}$-action at all the fixed points. We will see that all these data are isomorphic to those of known examples, $\widetilde{G}_{2}\left(\mathbb{R}^{n+2}\right)$ with $n \geq 2$ even, equipped with standard circle actions.
\end{abstract}

\section{INTRODUCTION}

Consider a circle action on a connected compact $2 n$-dimensional symplectic manifold $(M, \omega)$ with moment map $\phi$. By Morse theory, the fixed point set $M^{S^{1}}$ contains at least $n+1$ points. In [9], we study the case when $M^{S^{1}}$ consists of exactly $n+1$ isolated points. In this paper, we consider the case when $M^{S^{1}}$ consists of exactly $n+2$ isolated points, in which case, we call the action has almost minimal isolated fixed points. Such known examples are $\widetilde{G}_{2}\left(\mathbb{R}^{n+2}\right)$ with $n \geq 2$ even, see Example 2.1.

Let $(M, \omega)$ be a connected compact Hamiltonian $S^{1}$-manifold of dimension $2 n$ with almost minimal isolated fixed points, and let $\phi$ be the moment map. By Lemma 2.2, the fixed points, denoted $P_{0}, \cdots, P_{\frac{n}{2}-1}, P_{\frac{n}{2}}, P_{\left(\frac{n}{2}\right)^{\prime}}, P_{\frac{n}{2}+1}$, $\cdots, P_{n}$, can be labelled to satisfy

$$
\phi\left(P_{0}\right)<\cdots<\phi\left(P_{\frac{n}{2}-1}\right)<\phi\left(P_{\frac{n}{2}}\right) \leq \phi\left(P_{\left(\frac{n}{2}\right)^{\prime}}\right)<\phi\left(P_{\frac{n}{2}+1}\right)<\cdots<\phi\left(P_{n}\right) .
$$

In the statements of our main theorems below, we are referring to this order of moment map values of the fixed points.

For a symplectic $S^{1}$-manifold $M$ with isolated fixed points, in a neighborhood of each fixed point $P$, the $S^{1}$-action is equivalent to an $S^{1}$ linear

Key words and phrases. Symplectic manifold, Hamiltonian circle action, equivariant cohomology, Chern classes, Kähler manifold, biholomorphism, symplectomorphism.

2010 classification. 53D05, 53D20, 55N25, 57R20, 32H02.

The author is supported by the NSFC grant K110712116. 
action on $T_{P} M$, so there is a set of non-zero integers, called the weights of the $S^{1}$-action at the fixed point $P$.

Our main results are as follows.

Theorem 1. Let the circle act on a connected compact $2 n$-dimensional symplectic manifold $(M, \omega)$ with moment map $\phi: M \rightarrow \mathbb{R}$. Assume $[\omega]$ is a primitive integral class and the fixed point set $M^{S^{1}}$ consists of $n+2$ isolated points, denoted $M^{S^{1}}=\left\{P_{0}, \cdots, P_{\frac{n}{2}-1}, P_{\frac{n}{2}}, P_{\left(\frac{n}{2}\right)^{\prime}}, P_{\frac{n}{2}+1}, \cdots, P_{n}\right\}$. Then for any $i, j \in\left\{0, \cdots, \frac{n}{2},\left(\frac{n}{2}\right)^{\prime}, \cdots, n\right\}, \phi\left(P_{i}\right)-\phi\left(P_{j}\right) \in \mathbb{Z}$, and $c_{1}(M)=n[\omega]$ if and only if $\phi\left(P_{n-1}\right)-\phi\left(P_{0}\right)=\phi\left(P_{n}\right)-\phi\left(P_{1}\right)$ holds and this integer occurs as a weight of the $S^{1}$-action at some fixed point. In dimension 4 , for the "if" part to hold, the class $[\omega]$ needs to be chosen suitably.

Theorem 2. Let the circle act on a connected compact $2 n$-dimensional symplectic manifold $(M, \omega)$ with moment map $\phi: M \rightarrow \mathbb{R}$. Assume $M^{S^{1}}$ consists of $n+2$ isolated points, i.e., $M^{S^{1}}=\left\{P_{0}, \cdots, P_{\frac{n}{2}-1}, P_{\frac{n}{2}}, P_{\left(\frac{n}{2}\right)^{\prime}}, P_{\frac{n}{2}+1}, \cdots, P_{n}\right\}$. Then $n \geq 2$ must be even. Assume $[\omega]$ is an integral class. If $\phi\left(P_{n-1}\right)-$ $\phi\left(P_{0}\right)=\phi\left(P_{n}\right)-\phi\left(P_{1}\right)$ holds and this integer occurs as a weight of the $S^{1}$-action at some fixed point, then all the following are true.

(1) The integral cohomology ring of $M$ is isomorphic to that of $\widetilde{G}_{2}\left(\mathbb{R}^{n+2}\right)$.

(2) The total Chern class of $M$ is isomorphic to that of $\widetilde{G}_{2}\left(\mathbb{R}^{n+2}\right)$.

(3) The sets of weights of the $S^{1}$-action at all the fixed points on $M$ are isomorphic to those of a standard circle action on $\widetilde{G}_{2}\left(\mathbb{R}^{n+2}\right.$ ) (as in Example 2.1).

Theorem 2 follows from Propositions 4.5, 5.15 and 5.16

By [7, Prop. 4.2 and Sec. 5], if the manifold $M$ in Theorem 2 is Kähler, and the $S^{1}$-action is holomorphic, then $M$ is $S^{1}$-equivariantly biholomorphic and $S^{1}$-equivariantly symplectomorphic to $\widetilde{G}_{2}\left(\mathbb{R}^{n+2}\right)$ with $n \geq 2$ even.

In [4, Theorem 6.17], Hattori studies compact almost complex $S^{1}$-manifold $M$ of dimension $2 n$ with $n+2$ isolated fixed points. Under the assumption $c_{1}(M)=n x$, where $x \in H^{2}(M ; \mathbb{Z})$ is a generator, and under additional technical conditions, Hattori shows that the sets of weights at all the fixed points are isomorphic to those of a standard circle action on $\widetilde{G}_{2}\left(\mathbb{R}^{n+2}\right)$ with $n \geq 2$ even, and he obtains that $x^{n}[M]=2$. In our current work, for a compact symplectic Hamiltonian $S^{1}$-manifold $(M, \omega)$, we prove the equivalence of the condition $c_{1}(M)=n x$ and the particular weight as in Theorem 1, and using the particular weight as a starting point, we give methods to prove (1), (2) and (3) in Theorem 2

In [8], the author studies compact Hamiltonian $S^{1}$-manifolds with fixed point set consisting of two connected components and almost minimal in a certain sense. Recent related works on compact Hamiltonian $S^{1}$-manifolds with fixed point set minimal in a certain sense include [13, 12, 11, 7, 9, 10, 3. 5. 
The paper is organized as follows. In Section 2, we give examples of Hamiltonian $S^{1}$-manifolds with almost minimal isolated fixed points, prove and present some basic and key results. In Section 3, we prove Theorem 1 . In Section 4, we determine the sets of weights of the $S^{1}$-action at all the fixed points, proving (3) of Theorem 2, In Section 5. we determine the integral cohomology ring and the total Chern class of the manifold, proving (1) and (2) of Theorem 2 .

\section{EXAMPLES AND BASIC RESULTS}

In this section, we give examples, prove and present key basic results.

Example 2.1. Let $\widetilde{G}_{2}\left(\mathbb{R}^{n+2}\right)$ be the Grassmanian of oriented 2-planes in $\mathbb{R}^{n+2}$, with $n \geq 2$ even. This $2 n$-dimensional manifold naturally arises as a coadjoint orbit of $S O(n+2)$, hence it has a natural Kähler structure and a Hamiltonian $S O(n+2)$ action.

Consider the $S^{1} \subset S O(n+2)$ action on $\widetilde{G}_{2}\left(\mathbb{R}^{n+2}\right)$ induced by the $S^{1}$ action on $\mathbb{R}^{n+2}=\mathbb{C}^{\frac{n+2}{2}}$ given by

$$
\lambda \cdot\left(z_{0}, z_{1}, \cdots, z_{\frac{n}{2}}\right)=\left(\lambda^{b_{0}} z_{0}, \lambda^{b_{1}} z_{1}, \cdots, \lambda^{b_{\frac{n}{2}}} z_{\frac{n}{2}}\right),
$$

where the $b_{i}$ 's, with $i=0,1, \cdots, \frac{n}{2}$, are mutually distinct integers. This action has $n+2$ isolated fixed points, denoted

$$
P_{0}, P_{1}, \cdots, P_{\frac{n}{2}-1}, P_{\frac{n}{2}}, P_{\left(\frac{n}{2}\right)^{\prime}}, P_{\frac{n}{2}+1}, \cdots, P_{n},
$$

where for each $0 \leq i \leq \frac{n}{2}, P_{i}$ and $P_{n-i}$ are given by the plane $\left(0, \cdots, z_{i}, \cdots, 0\right)$ respectively with two different orientations. Here, we use the convention $n-\frac{n}{2}=\left(\frac{n}{2}\right)^{\prime}$. Let $\phi$ be the moment map of this $S^{1}$-action. Then the $\phi\left(P_{i}\right)^{\prime}$ 's are respectively

$$
-b_{0}, \cdots,-b_{\frac{n}{2}}, b_{\frac{n}{2}}, \cdots, b_{0}
$$

assuming in the order of nondecreasing. Note that if $b_{\frac{n}{2}} \neq 0$, then $\phi\left(P_{\frac{n}{2}}\right)<$ $\phi\left(P_{\left(\frac{n}{2}\right)^{\prime}}\right)$, otherwise, $\phi\left(P_{\frac{n}{2}}\right)=\phi\left(P_{\left(\frac{n}{2}\right)^{\prime}}\right)$. The set of weights of the $S^{1}$-action at any $P_{i}$, where $i \in\left\{0, \cdots, \frac{n}{2},\left(\frac{n}{2}\right)^{\prime}, \cdots, n\right\}$, is

$$
\left\{w_{i j}=\phi\left(P_{j}\right)-\phi\left(P_{i}\right)\right\}_{j \neq i, n-i}
$$

The ring $H^{*}\left(\widetilde{G}_{2}\left(\mathbb{R}^{n+2}\right) ; \mathbb{Z}\right)$ is generated by $x, y$ and $z$, where $\operatorname{deg}(x)=2$ and $\operatorname{deg}(y)=\operatorname{deg}(z)=n$. The relations are $x^{\frac{n}{2}}=y+z, x y=x z=$ $\frac{1}{2} x^{\frac{n}{2}+1}$, and $y^{2}=z^{2}=\frac{1+(-1)^{\frac{n}{2}}}{2} x^{\frac{n}{2}} y=\frac{1+(-1)^{\frac{n}{2}}}{2} x^{\frac{n}{2}} z$. The total Chern class $c\left(\widetilde{G}_{2}\left(\mathbb{R}^{n+2}\right)\right)=\frac{(1+x)^{n+2}}{1+2 x}$, in particular, $c_{1}\left(\widetilde{G}_{2}\left(\mathbb{R}^{n+2}\right)\right)=n x$.

Next, we prove a key basic lemma.

Lemma 2.2. Let the circle act on a connected compact $2 n$-dimensional symplectic manifold $(M, \omega)$ with moment map $\phi: M \rightarrow \mathbb{R}$. Assume $M^{S^{1}}$ consists of $n+2$ isolated points. Then $n$ must be even, and we can denote $M^{S^{1}}=\left\{P_{0}, \cdots, P_{\frac{n}{2}-1}, P_{\frac{n}{2}}, P_{\left(\frac{n}{2}\right)^{\prime}}, P_{\frac{n}{2}+1}, \cdots, P_{n}\right\}$, where the points can be 
labelled so that $P_{i}$ has Morse index $2 i$ for all $i$, and $P_{\left(\frac{n}{2}\right)^{\prime}}$ has Morse index $n$. Moreover, the cohomology groups of $M$ are

$$
H^{k}(M ; \mathbb{Z})= \begin{cases}\mathbb{Z}, & \text { if } k \text { is even, } 0 \leq k \leq 2 n, \text { and } k \neq n, \\ \mathbb{Z} \oplus \mathbb{Z}, & \text { if } k=n, \\ 0, & \text { if } k \text { is odd },\end{cases}
$$

and

$$
\phi\left(P_{0}\right)<\cdots<\phi\left(P_{\frac{n}{2}}\right) \leq \phi\left(P_{\left(\frac{n}{2}\right)^{\prime}}\right)<\phi\left(P_{\frac{n}{2}+1}\right)<\cdots<\phi\left(P_{n}\right) .
$$

Proof. Since $1,[\omega], \cdots,[\omega]^{n}$ are nonzero cohomology classes of $M$, the even Betti numbers $b_{2 i}(M) \geq 1$ for all $0 \leq 2 i \leq 2 n$. Since $\phi$ is a perfect Morse function, there is at least one fixed point, namely $P_{i}$, of index $2 i$ for each $0 \leq 2 i \leq 2 n$, which contributes to $b_{2 i}$. By assumption, there is a remaining fixed point, let $2 k$ be its Morse index. Since $b_{2 i}(M)=b_{2 n-2 i}(M)$ for all $0 \leq 2 i \leq 2 n$ by Poincaré duality, we must have $2 k=2 n-2 k$, which gives $2 k=n$, i.e., $n$ is even. We denote the remaining fixed point by $P_{\left(\frac{n}{2}\right)^{\prime}}$, which has Morse index $n$. By Morse theory, $M$ has a natural CW-structure, with cells given by the negative disk bundles of the fixed points. By cellular cohomology theory, the groups $H^{k}(M ; \mathbb{Z})$ are as claimed.

By [13, Lemma 3.1] (cited as Lemma 2.3 in [9]), for each $P_{i}, \operatorname{index}\left(P_{i}\right) \leq$ $2 l$, where $l$ is the number of fixed points below $P_{i}$, we obtain $\phi\left(P_{0}\right)<$ $\phi\left(P_{1}\right)<\cdots<\phi\left(P_{\frac{n}{2}}\right) \leq \phi\left(P_{\left(\frac{n}{2}\right)^{\prime}}\right)$. Similarly, using $-\phi$, we obtain $-\phi\left(P_{n}\right)<$ $-\phi\left(P_{n-1}\right)<\cdots<-\phi\left(P_{\left(\frac{n}{2}\right)^{\prime}}\right) \leq-\phi\left(P_{\frac{n}{2}}\right)$. The two inequalities give (2.3).

Remark 2.4. From now on, when we use the notation

$$
M^{S^{1}}=\left\{P_{0}, \cdots, P_{\frac{n}{2}-1}, P_{\frac{n}{2}}, P_{\left(\frac{n}{2}\right)^{\prime}}, P_{\frac{n}{2}+1}, \cdots, P_{n}\right\},
$$

we mean that each point has the Morse index as in Lemma 2.2, and these points satisfy (2.3). We will use these facts tacitly.

Next, we get a basis of the equivariant and ordinary cohomology of the manifold. When we speak about $S^{1}$-equivariant cohomology, we use $t \in H^{2}\left(\mathbb{C P}^{\infty}, \mathbb{Z}\right)$ to denote a generator. For basic material in equivariant cohomology, we refer to $[9]$.

Proposition 2.5. Let $(M, \omega)$ be a connected compact $2 n$-dimensional Hamiltonian $S^{1}$-manifold with moment $\operatorname{map} \phi: M \rightarrow \mathbb{R}$. Assume

$$
M^{S^{1}}=\left\{P_{0}, \cdots, P_{\frac{n}{2}-1}, P_{\frac{n}{2}}, P_{\left(\frac{n}{2}\right)^{\prime}}, P_{\frac{n}{2}+1}, \cdots, P_{n}\right\} .
$$

Let $I=\left\{0, \cdots, \frac{n}{2},\left(\frac{n}{2}\right)^{\prime}, \cdots, n\right\}$. Then as an $H^{*}\left(\mathbb{C P}^{\infty} ; \mathbb{Z}\right)$-module, $H_{S^{1}}^{*}(M ; \mathbb{Z})$ has a basis $\left\{\widetilde{\alpha}_{i} \mid i \in I\right\}$ such that for any $i \in I$,

$$
\left.\widetilde{\alpha}_{i}\right|_{P_{i}}=\Lambda_{i}^{-} t^{i},\left.\quad \widetilde{\alpha}_{i}\right|_{P_{j}}=0, \forall P_{j} \text { with } \operatorname{index}\left(P_{j}\right) \leq \operatorname{index}\left(P_{i}\right),
$$

where $\Lambda_{i}^{-}$is the product of the negative weights at $P_{i}$. Moreover, $\left\{\alpha_{i}=\right.$ $\left.r\left(\widetilde{\alpha}_{i}\right) \mid i \in I\right\}$ is a basis for $H^{*}(M ; \mathbb{Z})$, where $r: H_{S^{1}}^{*}(M ; \mathbb{Z}) \rightarrow H^{*}(M ; \mathbb{Z})$ is the natural restriction. 
Proof. Using standard argument in equivariant cohomology, we get the basis of $H_{S^{1}}^{*}(M ; \mathbb{Z})$ satisfying the other conditions except that $\left.\widetilde{\alpha}_{\frac{n}{2}}\right|_{P_{\left(\frac{n}{2}\right)^{\prime}}}=0$. If $\left.\widetilde{\alpha}_{\frac{n}{2}}\right|_{P_{\left(\frac{n}{2}\right)^{\prime}}}=\left.a \widetilde{\alpha}_{\left(\frac{n}{2}\right)^{\prime}}\right|_{P_{\left(\frac{n}{2}\right)^{\prime}}} \neq 0$, where $a \in \mathbb{Z}$, then replace $\widetilde{\alpha}_{\frac{n}{2}}$ by $\widetilde{\alpha}_{\frac{n}{2}}-a \widetilde{\alpha}_{\left(\frac{n}{2}\right)^{\prime}}$, still denoting it $\widetilde{\alpha} \frac{n}{2}$.

Since the fixed points are isolated, hence has no torsion cohomology, the restriction map $r$ is onto ([11, Sec. 2]), so $\left\{\alpha_{i}=r\left(\widetilde{\alpha}_{i}\right) \mid i \in I\right\}$ is a basis for $H^{*}(M ; \mathbb{Z})$.

Corollary 2.6. Let $(M, \omega)$ be a connected compact $2 n$-dimensional Hamiltonian $S^{1}$-manifold with moment map $\phi: M \rightarrow \mathbb{R}$. Assume

$$
M^{S^{1}}=\left\{P_{0}, \cdots, P_{\frac{n}{2}-1}, P_{\frac{n}{2}}, P_{\left(\frac{n}{2}\right)^{\prime}}, P_{\frac{n}{2}+1}, \cdots, P_{n}\right\} .
$$

If $\widetilde{\alpha} \in H_{S^{1}}^{2 k}(M ; \mathbb{Z})$ is a class such that $\left.\widetilde{\alpha}\right|_{P_{i}}=0$ for all $P_{i}$ 's with $\phi\left(P_{i}\right)<a$, then

$$
\widetilde{\alpha}=\sum_{i} a_{i} \widetilde{\alpha}_{i},
$$

where the sum is over the indices $i$ 's such that $\phi\left(P_{i}\right) \geq a$ and $\operatorname{deg}\left(\widetilde{\alpha}_{i}\right) \leq 2 k$, and $a_{i} \in H^{*}\left(\mathbb{C P} \mathbb{P}^{\infty} ; \mathbb{Z}\right)$. Here, $i \in I=\left\{0, \cdots, \frac{n}{2},\left(\frac{n}{2}\right)^{\prime}, \cdots, n\right\}$.

Next, we state some known results which we will use in the next sections.

Lemma 2.7. [9, Lemmas 2.1 and 2.2] Let the circle act on a connected compact symplectic manifold $(M, \omega)$ with moment map $\phi: M \rightarrow \mathbb{R}$. Assume the fixed points are isolated. Then there exists $\widetilde{u}=[\omega-\phi t] \in H_{S^{1}}^{2}(M ; \mathbb{R})$ such that for any fixed point $P$,

$$
\left.\widetilde{u}\right|_{P}=-\phi(P) t .
$$

If $[\omega]$ is an integral class, then $\widetilde{u}$ is an integral class. In this case, for any two fixed points $P$ and $Q, \phi(P)-\phi(Q) \in \mathbb{Z}$. If $\mathbb{Z}_{k}$ is the stabilizer group of some point on $M$, then for any two fixed points $P$ and $Q$ on the same connected component of $M^{\mathbb{Z}_{k}}$, we have $k \mid(\phi(P)-\phi(Q))$.

In Lemma 2.7, $M^{\mathbb{Z}_{k}}$ is the $\mathbb{Z}_{k}$-isotropy submanifold, i.e., the set of points pointwise fixed by $\mathbb{Z}_{k}$ but not pointwise fixed by $S^{1}$.

By Lemma 2.7, if $[\omega]$ is an integral class, then any weight of the $S^{1}$-action is no larger than the length of the interval $\phi(M)$.

Theorem 3. 1, 2] Let the circle act on a compact oriented manifold $M$. Assume the fixed points are isolated. Fix a class $\alpha \in H_{S^{1}}^{*}(M ; \mathbb{Q})$. Then as elements of $\mathbb{Q}(t)$,

$$
\int_{M} \alpha=\sum_{P \subset M^{S^{1}}} \frac{\left.\alpha\right|_{P}}{e^{S^{1}}\left(N_{P}\right)},
$$

where the sum is over all the fixed points, and $e^{S^{1}}\left(N_{P}\right)$ is the equivariant Euler class of the normal bundle to $P$. 
Lemma 2.8. [13, Lemma 2.6] Let the circle act on a compact symplectic manifold $(M, \omega)$. Let $p$ and $q$ be fixed points which lie on the same connected component of $M^{\mathbb{Z}_{k}}$ for some $k>1$. Then the $S^{1}$ weights at $p$ and $q$ are equal modulo $k$.

\section{ON THE FIRST CHERN Class OF THE MANIFOLD - THE PROOF OF}

THEOREM 1

In this section, we prove Theorem 1,

In a symplectic $S^{1}$-manifold $(M, \omega)$ with isolated fixed points, if $w>0$ is a weight of the $S^{1}$-action at a fixed point $P,-w$ is a weight of the $S^{1}$-action at a fixed point $Q$, and $P$ and $Q$ are on the same connected component of $M^{\mathbb{Z}_{w}}$, then we say that there is a weight $w$ from $P$ to $Q$, or $w$ is a weight from $P$ to $Q$. When the signs of $w$ at $P$ and at $Q$ are clear, we also say that there is a weight $\pm w$ between $P$ and $Q$. It is known (see [4] for example) that the set $W^{+}$of all the positive weights and the set $W^{-}$of all the negative weights at all the fixed points satisfy $W^{-}=-W^{+}$. We will use this fact tacitly.

The following Lemma 3.1 is a consequence of Lemmas 4.2 and 4.4 in [9].

Lemma 3.1. Let the circle act on a connected compact symplectic manifold $(M, \omega)$ with moment map $\phi: M \rightarrow \mathbb{R}$. Assume

$$
M^{S^{1}}=\left\{P_{0}, \cdots, P_{\frac{n}{2}-1}, P_{\frac{n}{2}}, P_{\left(\frac{n}{2}\right)^{\prime}}, P_{\frac{n}{2}+1}, \cdots, P_{n}\right\} .
$$

Let $w>0$ be the largest in the set of all the weights of the $S^{1}$-action at all the fixed points. Then there are $P_{i}$ and $P_{j}$ with $\operatorname{index}\left(P_{i}\right) \leq \operatorname{index}\left(P_{j}\right)$, such that $w$ is a weight from $P_{i}$ to $P_{j}$. Here, $i, j \in I=\left\{0, \cdots, \frac{n}{2},\left(\frac{n}{2}\right)^{\prime}, \cdots, n\right\}$. If $c_{1}(M)=k[\omega]$, then $\frac{1}{2} \operatorname{index}\left(P_{j}\right)-\frac{1}{2} \operatorname{index}\left(P_{i}\right)+1=k \frac{\phi\left(P_{j}\right)-\phi\left(P_{i}\right)}{w}$.

We will use Lemma 3.2 in the proof of Lemma 3.3.

Lemma 3.2. Let the circle act on a connected compact symplectic manifold $(M, \omega)$ with moment map $\phi: M \rightarrow \mathbb{R}$. Assume $M^{S^{1}}$ consists of isolated points. Then for any index 2 fixed point, there is a weight between it and a fixed point below it (relative to the value of $\phi$ ). Similarly, for any coindex 2 fixed point, there is a weight between it and a fixed point above it.

Proof. Let $P \in M^{S^{1}}$ be of index 2 and $-w$ be the negative weight at $P$. Since $P$ has only one negative weight, the connected component $C$ of $M^{\mathbb{Z}_{w}}$ containing $P$ has $P$ as an index 2 fixed point. Since $C$ is compact and symplectic, it contains a unique fixed point $Q$ as the minimum of $\left.\phi\right|_{C}$, so $\phi(Q)<\phi(P)$. Note that $w$ is the smallest positive weight on $C$ (other weights on $C$ must be multiples of $w$ ), using [5, Proposition 3] (cited as Lemma 5.6 in [9]) on $C$, we get that $w$ is a positive weight at $Q$. If $w=1$, we regard $C=M$. The other claim follows similarly by using $-\phi$.

To prove Theorem 1, note that when $\operatorname{dim}(M)=4, \operatorname{dim} H^{2}(M)$ is 2 . In this case, we need to choose the class $[\omega]$ suitably so that $c_{1}(M)$ is a multiple 
of $[\omega]$. We consider this case separately, at the same time, we obtain the sets of weights at all the fixed points.

Lemma 3.3. Let the circle act on a connected compact 4-dimensional symplectic manifold $(M, \omega)$ with moment map $\phi: M \rightarrow \mathbb{R}$. Assume $[\omega]$ is an integral class and $M^{S^{1}}=\left\{P_{0}, P_{1}, P_{1^{\prime}}, P_{2}\right\}$. Assume $\phi\left(P_{1^{\prime}}\right)-\phi\left(P_{0}\right)=$ $\phi\left(P_{2}\right)-\phi\left(P_{1}\right)$ is a weight of the $S^{1}$-action at some fixed point. Then

$$
\phi\left(P_{1}\right)-\phi\left(P_{0}\right)=\phi\left(P_{2}\right)-\phi\left(P_{1^{\prime}}\right)
$$

and $[\omega]$ is primitive integral. Assume furthermore that $\left[\left.\omega\right|_{S_{1}}\right]$ is primitive integral, where $S_{1}$ is an invariant gradient sphere for any invariant metric from $P_{1}$ to $P_{0}$. Then the set of weights at any $P_{i}$ is

$$
\left\{w_{i j}=\phi\left(P_{j}\right)-\phi\left(P_{i}\right)\right\}_{j \neq i, 2-i}
$$

where $i, j \in\left\{0,1,1^{\prime}, 2\right\}$, and we use the convention $1+1^{\prime}=2$; moreover, $c_{1}(M)=2[\omega]$.

Proof. The equality $\phi\left(P_{1^{\prime}}\right)-\phi\left(P_{0}\right)=\phi\left(P_{2}\right)-\phi\left(P_{1}\right)$ implies (3.4).

Denote $w_{01^{\prime}}=\phi\left(P_{1^{\prime}}\right)-\phi\left(P_{0}\right)$. If $\phi\left(P_{1}\right)<\phi\left(P_{1^{\prime}}\right)$, then $w_{01^{\prime}}$ can only divide $\phi\left(P_{1^{\prime}}\right)-\phi\left(P_{0}\right)$, hence it can only be a weight between $P_{0}$ and $P_{1^{\prime}}$. If $\phi\left(P_{1}\right)=\phi\left(P_{1^{\prime}}\right)$, then by Lemma 3.2. each of $P_{1}$ and $P_{1^{\prime}}$ has a weight with $P_{0}$, hence in this case $w_{01^{\prime}}$ is also a weight between $P_{0}$ and $P_{1^{\prime}}$ (not between $P_{0}$ and $P_{2}$, since there can only be two weights at $P_{0}$ ). For any invariant metric, let $S_{1^{\prime}}$ be the gradient sphere from $P_{1^{\prime}}$ to $P_{0}$ (if $\gamma$ is the gradient line from $P_{1^{\prime}}$ to $P_{0}$, then $S^{1} \cdot \gamma$ is the gradient sphere), then

$$
\int_{S_{1^{\prime}}}[\omega]=\frac{\phi\left(P_{1^{\prime}}\right)-\phi\left(P_{0}\right)}{w_{01^{\prime}}}=1 .
$$

This means that $\left[\left.\omega\right|_{S_{1^{\prime}}}\right]$ is primitive integral, hence $[\omega]$ is primitive integral. Let the weight between $P_{1}$ and $P_{0}$ be $w$. By assumption, $\left[\left.\omega\right|_{S_{1}}\right]$ is primitive integral, similar to the above, we obtain that $|w|=\phi\left(P_{1}\right)-\phi\left(P_{0}\right)$. Denote $w_{01}=\phi\left(P_{1}\right)-\phi\left(P_{0}\right)$. By symmetry, or by using $-\phi$, similarly, we know that $w_{12}=\phi\left(P_{2}\right)-\phi\left(P_{1}\right)$ is a weight between $P_{1}$ and $P_{2}$, and $w_{1^{\prime} 2}=\phi\left(P_{2}\right)-\phi\left(P_{1^{\prime}}\right)$ is a weight between $P_{1^{\prime}}$ and $P_{2}$. The claim (3.5) follows.

Now consider the basis element $\widetilde{\alpha}_{1}$ and $\widetilde{\alpha}_{1^{\prime}}$ of $H_{S^{1}}^{2}(M ; \mathbb{Z})$ in Proposition 2.5. It is easy to check, by using (3.5) for $P_{1}$ and $P_{1^{\prime}}$, that the restrictions of the degree 2 classes $\widetilde{u}+\phi\left(P_{0}\right) t$ and $\widetilde{\alpha}_{1}+\widetilde{\alpha}_{1^{\prime}}$ to the index 0 and index 2 fixed points $P_{0}, P_{1}$ and $P_{1^{\prime}}$ are equal. Hence

$$
\widetilde{u}+\phi\left(P_{0}\right) t=\widetilde{\alpha}_{1}+\widetilde{\alpha}_{1^{\prime}} .
$$

Restricting it to ordinary cohomology, we obtain $[\omega]=\alpha_{1}+\alpha_{1^{\prime}}$. By the same argument, we obtain

$$
c_{1}^{S^{1}}(M)=2 \widetilde{\alpha}_{1}+2 \widetilde{\alpha}_{1^{\prime}}+\Gamma_{0} t
$$

Restricting this to ordinary cohomology, we obtain $c_{1}(M)=2\left(\alpha_{1}+\alpha_{1^{\prime}}\right)$. So the claim $c_{1}(M)=2[\omega]$ follows. 
To prove Theorem 1, let us first show that there is no weight larger than the integer $\phi\left(P_{n-1}\right)-\phi\left(P_{0}\right)=\phi\left(P_{n}\right)-\phi\left(P_{1}\right)$.

Proposition 3.6. Let the circle act on a connected compact symplectic manifold $(M, \omega)$ with moment map $\phi: M \rightarrow \mathbb{R}$. Assume $[\omega]$ is an integral class and $M^{S^{1}}=\left\{P_{0}, \cdots, P_{\frac{n}{2}-1}, P_{\frac{n}{2}}, P_{\left(\frac{n}{2}\right)^{\prime}}, P_{\frac{n}{2}+1}, \cdots, P_{n}\right\}$. Then the integer $\phi\left(P_{n}\right)-\phi\left(P_{0}\right)$ cannot be a weight at any fixed point.

Proof. Let $I=\left\{0, \cdots, \frac{n}{2},\left(\frac{n}{2}\right)^{\prime}, \cdots, n\right\}$. By Lemma 2.7. for any $i, j \in I$, $\phi\left(P_{i}\right)-\phi\left(P_{j}\right) \in \mathbb{Z}$. Suppose $w_{0 n}=\phi\left(P_{n}\right)-\phi\left(P_{0}\right)$ is a weight at some fixed point. Since it only divides $\phi\left(P_{n}\right)-\phi\left(P_{0}\right)$, it can only be a weight between $P_{0}$ and $P_{n}$, and it can only have multiplicity 1 . Similar to the proof of $[9$, Lemma 5.7], using

$$
\left\{\text { weights at } P_{0}\right\}=\left\{\text { weights at } P_{n}\right\} \bmod w_{0 n},
$$

we obtain that the possible weights at $P_{0}$ and at $P_{n}$ are respectively in the sets

$$
\left\{\phi\left(P_{j}\right)-\phi\left(P_{0}\right)\right\}_{j \in I, j \neq 0}, \text { and }\left\{\phi\left(P_{j}\right)-\phi\left(P_{n}\right)\right\}_{j \in I, j \neq n} .
$$

Moreover, in the following two cases, $\phi\left(P_{j}\right)-\phi\left(P_{0}\right)$ is a weight at $P_{0}$ if and only if $\phi\left(P_{j}\right)-\phi\left(P_{n}\right)$ is a weight at $P_{n}$.

(1) $\phi\left(P_{\frac{n}{2}}\right)<\phi\left(P_{\left(\frac{n}{2}\right)^{\prime}}\right)$, and $j \neq 0, n$.

(2) $\phi\left(P_{\frac{n}{2}}\right)=\phi\left(P_{\left(\frac{n}{2}\right)^{\prime}}\right)$, and $j \neq 0, n, \frac{n}{2},\left(\frac{n}{2}\right)^{\prime}$.

Combining with a slight modification of [9, Lemma 5.1], we can see that any weight occurs with multiplicity 1 . Since there are $n$ number of weights at $P_{0}$ and at $P_{n}$, and there are $n+1$ number of elements in each set in (3.7), there exists $k \neq 0, n$ (also $k \neq 1, n-1$ if we use Lemma 3.2) such that $\phi\left(P_{k}\right)-\phi\left(P_{0}\right)$ does not occur as a weight at $P_{0}$. By symmetry, $\phi\left(P_{n-k}\right)-\phi\left(P_{n}\right)$ does not occur as a weight at $P_{n}$. If $\phi\left(P_{\frac{n}{2}}\right)<\phi\left(P_{\left(\frac{n}{2}\right)^{\prime}}\right)$, or if $\phi\left(P_{\frac{n}{2}}\right)=\phi\left(P_{\left(\frac{n}{2}\right)^{\prime}}\right)$ and $k \neq \frac{n}{2},\left(\frac{n}{2}\right)^{\prime}$, then by the fact above, $\phi\left(P_{n-k}\right)-\phi\left(P_{0}\right)$ is also not a weight at $P_{0}$ (similarly $\phi\left(P_{k}\right)-\phi\left(P_{n}\right)$ is not a weight at $\left.P_{n}\right)$, so $P_{0}$ (and $P_{n}$ ) has less than $n$ number of weights, a contradiction. Now assume $\phi\left(P_{\frac{n}{2}}\right)=\phi\left(P_{\left(\frac{n}{2}\right)^{\prime}}\right)$, and $k=\left(\frac{n}{2}\right)^{\prime}$ (similarly for $\left.k=\frac{n}{2}\right)$. It is conceivable that $\phi\left(P_{\frac{n}{2}}\right)-\phi\left(P_{0}\right)$ occurs at $P_{0}$ and $\phi\left(P_{\left(\frac{n}{2}\right)^{\prime}}\right)-\phi\left(P_{0}\right)$ does not occur at $P_{0}$, and $\phi\left(P_{\left(\frac{n}{2}\right)^{\prime}}\right)-\phi\left(P_{n}\right)$ occurs at $P_{n}$ and $\phi\left(P_{\frac{n}{2}}\right)-\phi\left(P_{n}\right)$ does not occur at $P_{n}$. We claim that this cannot happen. We look at it as follows. By symmetry, we may normalize $[\omega]$ and $\phi$ such that $\phi\left(P_{\frac{n}{2}}\right)-\phi\left(P_{0}\right)=\phi\left(P_{n}\right)-\phi\left(P_{\frac{n}{2}}\right)$. Denote $w=\phi\left(P_{\frac{n}{2}}\right)-\phi\left(P_{0}\right)=$ $\phi\left(P_{n}\right)-\phi\left(P_{\frac{n}{2}}\right)$. Then $w_{0 n}=2 w$. Note that $w$, as a weight at $P_{0}$, can only divide $\phi\left(P_{\frac{n}{2}}\right)-\phi\left(P_{0}\right)$ and $\phi\left(P_{n}\right)-\phi\left(P_{0}\right)$. By [9, Lemma 5.5], $w$ is a weight between $P_{0}$ and $P_{\frac{n}{2}}$, and this number is a weight between $P_{\left(\frac{n}{2}\right)^{\prime}}$ and $P_{n}$. The isotropy submanifold $M^{\mathbb{Z}_{w}}$ containing $P_{0}$ contains also $P_{\frac{n}{2}}, P_{n}$ and $P_{\left(\frac{n}{2}\right)^{\prime}}$. It is 4-dimensional with exactly these 4 fixed points, there is a weight $w_{0 n}$ between $P_{0}$ and $P_{n}$, and there is no weight between $P_{\left(\frac{n}{2}\right)^{\prime}}$ and $P_{0}$. By 
Lemma 3.2 (used on $M^{\mathbb{Z}_{w}}$ ), the weights structure on this submanifold is not possible.

Proof of Theorem 11. First, by Lemma 2.7, we have $\phi\left(P_{i}\right)-\phi\left(P_{j}\right) \in \mathbb{Z}$ for any $i, j \in\left\{0, \cdots, \frac{n}{2},\left(\frac{n}{2}\right)^{\prime}, \cdots, n\right\}$.

If $\operatorname{dim}(M)=2 n>4$, by Lemma $\left[2.2, H^{2}(M ; \mathbb{Z})=\mathbb{Z}\right.$. So $c_{1}(M)=k[\omega]$ for some $k \in \mathbb{Z}$. Let $w>0$ be the largest weight of the action at all the fixed points. By Lemma 3.1, $w$ is a weight from $P_{i}$ to $P_{j}$ with $\operatorname{index}\left(P_{i}\right) \leq$ index $\left(P_{j}\right)$. Then $w \mid\left(\phi\left(P_{j}\right)-\phi\left(P_{i}\right)\right)$ by Lemma 2.7. By Lemma 3.1, $k=n$ implies that $w=\phi\left(P_{n-1}\right)-\phi\left(P_{0}\right)=\phi\left(P_{n}\right)-\phi\left(P_{1}\right)$. Conversely, assume $\phi\left(P_{n-1}\right)-\phi\left(P_{0}\right)=\phi\left(P_{n}\right)-\phi\left(P_{1}\right)$ holds and this integer occurs as a weight, by Proposition 3.6, this integer is the largest weight; since it only divides $\phi\left(P_{n-1}\right)-\phi\left(P_{0}\right)$ and $\phi\left(P_{n}\right)-\phi\left(P_{1}\right)$, it is a weight between $P_{0}$ and $P_{n-1}$ (and is also a weight between $P_{1}$ and $\left.P_{n}\right)$. By Lemma 3.1, $k=n$.

Now consider $\operatorname{dim}(M)=4$. If $c_{1}(M)=2[\omega]$, then the same argument as above yields that $\phi\left(P_{1^{\prime}}\right)-\phi\left(P_{0}\right)=\phi\left(P_{2}\right)-\phi\left(P_{1}\right)$ is the largest weight. For the converse, we do not know that $c_{1}(M)$ is a multiple of $[\omega]$; the converse is by Lemma 3.3 .

\section{FROM THE NAMED WEIGHT TO ALL THE WEIGHTS}

In this section, using the given weight, we find the sets of weights at all the fixed points, proving (3) of Theorem 2.

For convenience, we state the following lemma.

Lemma 4.1. 9, Lemma 4.1] Let the circle act on a connected compact symplectic manifold $(M, \omega)$ with moment map $\phi: M \rightarrow \mathbb{R}$. If $c_{1}(M)=k[\omega]$, then for any two fixed point set components $F$ and $F^{\prime}$, we have $\Gamma_{F}-\Gamma_{F^{\prime}}=$ $k\left(\phi\left(F^{\prime}\right)-\phi(F)\right)$, where $\Gamma_{F}$ and $\Gamma_{F^{\prime}}$ are respectively the sums of the weights at $F$ and $F^{\prime}$.

We first find the sets of weights at $P_{0}, P_{1}, P_{n-1}$ and $P_{n}$.

Lemma 4.2. Let the circle act on a connected compact $2 n$-dimensional symplectic manifold $(M, \omega)$ with moment map $\phi: M \rightarrow \mathbb{R}$. Assume $[\omega]$ is an integral class and $M^{S^{1}}=\left\{P_{0}, \cdots, P_{\frac{n}{2}-1}, P_{\frac{n}{2}}, P_{\left(\frac{n}{2}\right)^{\prime}}, P_{\frac{n}{2}+1} \cdots, P_{n}\right\}$. Assume $\phi\left(P_{n-1}\right)-\phi\left(P_{0}\right)=\phi\left(P_{n}\right)-\phi\left(P_{1}\right)$ occurs as a weight of the $S^{1}$-action at some fixed point. Then

$$
\phi\left(P_{1}\right)-\phi\left(P_{0}\right)=\phi\left(P_{n}\right)-\phi\left(P_{n-1}\right),
$$

$[\omega]$ is primitive integral, and for $i=0,1, n-1, n$, the set of weights at $P_{i}$ is

$$
\left\{w_{i j}=\phi\left(P_{j}\right)-\phi\left(P_{i}\right)\right\}_{j \neq i, n-i} .
$$

Proof. Lemma 3.3 gives the claims for $n=2$. So we assume now $n>2$. The fact $\phi\left(P_{n-1}\right)-\phi\left(P_{0}\right)=\phi\left(P_{n}\right)-\phi\left(P_{1}\right)$ implies (4.3). Let $w_{0, n-1}=$ $\phi\left(P_{n-1}\right)-\phi\left(P_{0}\right)$. Since $w_{0, n-1}$ only divides $\phi\left(P_{n-1}\right)-\phi\left(P_{0}\right), w_{0, n-1}$ can 
only be a weight between $P_{0}$ and $P_{n-1}$ (and between $P_{n}$ and $P_{1}$ ). As a weight between any pair of points, it can only have multiplicity 1 .

For the following steps, we proceed similarly as in the proof of Lemma 5.16 in $[9]$. First we observe that the integration of $[\omega]$ on the isotropy sphere $M^{\mathbb{Z}_{w_{0, n-1}}}$ is 1 , so we get that $[\omega]$ is primitive integral. Since $n>2$, there is a unique index 2 fixed point $P_{1}$ and a unique coindex 2 fixed point $P_{n-1}$; the proof of [9, Lemma 5.15] still works, and we get that $w_{01}=\phi\left(P_{1}\right)-\phi\left(P_{0}\right)$ is a weight between $P_{0}$ and $P_{1}$, and $w_{n-1, n}=\phi\left(P_{n}\right)-\phi\left(P_{n-1}\right)$ is a weight between $P_{n-1}$ and $P_{n}$. By Proposition 3.6, $\phi\left(P_{n}\right)-\phi\left(P_{0}\right)$ is not a weight, so $w_{0, n-1}$ is the largest weight. As in the proof of [9, Lemma 5.16], we use

$$
\left\{\text { weights at } P_{0}\right\}=\left\{\text { weights at } P_{n-1}\right\} \quad \bmod w_{0, n-1}
$$

to get the following possibilities about the weights at $P_{0}$ and $P_{n-1}$ :

(1) For $j \in\left\{1, \cdots, \frac{n}{2},\left(\frac{n}{2}\right)^{\prime}, \cdots, n-2\right\}$, there is a weight $w_{0 j}=\phi\left(P_{j}\right)-$ $\phi\left(P_{0}\right)$ between $P_{0}$ and some $P_{m}$ with $m \in\left\{2, \cdots, \frac{n}{2},\left(\frac{n}{2}\right)^{\prime}, \cdots, n-\right.$ $2\}$, or between $P_{0}$ and $P_{n}$, and correspondingly, there is a weight $w_{n-1, j}=\phi\left(P_{j}\right)-\phi\left(P_{n-1}\right)$ between $P_{n-1}$ and some $P_{l}$ with $l \in$ $\left\{1, \cdots, \frac{n}{2},\left(\frac{n}{2}\right)^{\prime}, \cdots, n-2\right\}$. (The $w_{01}$ here is different from the one above between $P_{0}$ and $P_{1}$, or one may view it as it appeared with multiplicities.)

(2) $\bar{w}_{0 n}=\frac{1}{2}\left(\phi\left(P_{n}\right)-\phi\left(P_{0}\right)\right)$ is a weight between $P_{0}$ and $P_{n}$ with multiplicity 1 , and correspondingly, $\bar{w}_{n-1,1}=\frac{1}{2}\left(\phi\left(P_{1}\right)-\phi\left(P_{n-1}\right)\right)$ is a weight between $P_{n-1}$ and $P_{1}$ with multiplicity 1 .

We have 3 claims about these possibilities:

Claim 1: For $j \in\left\{1, \cdots, \frac{n}{2},\left(\frac{n}{2}\right)^{\prime}, \cdots, n-2\right\}$, if $w_{n-1, j}=\phi\left(P_{j}\right)-\phi\left(P_{n-1}\right)$ appears as a weight at $P_{n-1}$, then it has multiplicity 1 .

Claim 2: $w_{n-1,1}$ and $\bar{w}_{n-1,1}$ cannot appear at the same time.

Claim 3: Neither $w_{n-1,1}$ nor $\bar{w}_{n-1,1}$ can appear.

The proofs of Claims 1 and 2 are the same as in the proof of [9, Lemma 5.16] (Lemma 5.1 in [9] might be used with very minor modification when the two index $n$ fixed points have equal moment map values). Now we prove Claim 3. First assume $w_{n-1,1}$ appears. Then by Claims 1 and 2, $w_{n-1,1}$ has multiplicity 1 and $\bar{w}_{n-1,1}$ does not appear, hence (2) does not occur. Since there are $n-1$ number of negative weights at $P_{n-1}$, and there are $n$ number of fixed points below $P_{n-1}$, by (1) and Claim 1, there exists one $k \in\left\{2, \cdots, \frac{n}{2},\left(\frac{n}{2}\right)^{\prime}, \cdots, n-2\right\}$ such that $w_{n-1, k}=\phi\left(P_{k}\right)-\phi\left(P_{n-1}\right)$ is not a weight at $P_{n-1}$. By symmetry, or similarly by using $-\phi$, we get that $w_{1, n-k}=\phi\left(P_{n-k}\right)-\phi\left(P_{1}\right)$ is not a (positive) weight at $P_{1}$. Then we can write down the sets of weights at $P_{n-1}$ and $P_{1}$ :

$$
\begin{gathered}
\left\{w_{n-1, j}=\phi\left(P_{j}\right)-\phi\left(P_{n-1}\right)\right\}_{j \neq n-1, k}, \\
\left\{w_{1 j}=\phi\left(P_{j}\right)-\phi\left(P_{1}\right)\right\}_{j \neq 1, n-k}, \text { where } w_{1, n-1}=-w_{n-1,1},
\end{gathered}
$$


for some $k \in\left\{2, \cdots, \frac{n}{2},\left(\frac{n}{2}\right)^{\prime}, \cdots, n-2\right\}$. (Here $n-\frac{n}{2}=\left(\frac{n}{2}\right)^{\prime}$.) We compute

$$
\Gamma_{1}-\Gamma_{n-1}=\sum w_{1 j}-\sum w_{n-1, j} \neq n\left(\phi\left(P_{n-1}\right)-\phi\left(P_{1}\right)\right),
$$

which contradicts to Lemma 4.1 and Theorem 1 1 . Hence $w_{n-1,1}$ does not appear. (We could also use $P_{0}$ and $P_{n-1}$, or $P_{0}$ and $P_{n}$ to do the argument above.) Next, assume $\bar{w}_{n-1,1}$ appears, i.e., (2) occurs. Then $w_{n-1,1}$ does not appear (the corresponding $w_{01}$ in (1) does not appear). Similar to the above, let us write down the sets of weights at $P_{0}$ and $P_{n}$ :

$$
\begin{gathered}
\left\{w_{0 j}=\phi\left(P_{j}\right)-\phi\left(P_{0}\right)\right\}_{j \neq 0, n, k} \cup \bar{w}_{0 n}, \\
\left\{w_{n j}=\phi\left(P_{j}\right)-\phi\left(P_{n}\right)\right\}_{j \neq n, 0, n-k} \cup \bar{w}_{n 0}, \text { where } \bar{w}_{n 0}=-\bar{w}_{0 n},
\end{gathered}
$$

for some $k \in\left\{2, \cdots, \frac{n}{2},\left(\frac{n}{2}\right)^{\prime}, \cdots, n-2\right\}$. If $\phi\left(P_{\frac{n}{2}}\right)<\phi\left(P_{\left(\frac{n}{2}\right)^{\prime}}\right)$, or if $\phi\left(P_{\frac{n}{2}}\right)=$ $\phi\left(P_{\left(\frac{n}{2}\right)^{\prime}}\right)$ and $k \neq \frac{n}{2},\left(\frac{n}{2}\right)^{\prime}$, the same argument as above gives a contradiction. Now assume $\phi\left(P_{\frac{n}{2}}\right)=\phi\left(P_{\left(\frac{n}{2}\right)^{\prime}}\right)$ and $k=\left(\frac{n}{2}\right)^{\prime}$ ( or $\left.k=\frac{n}{2}\right)$. We argue similarly as in the last part of the proof of Proposition 3.6 to get a contradiction by looking at the weights structure on a 4-dimensional isotropy submanifold containing $P_{0}, P_{\frac{n}{2}}, P_{\left(\frac{n}{2}\right)^{\prime}}$ and $P_{n}$. By all the facts above, the claims on the weights follow.

Next, we find the sets of weights at all the fixed points.

Proposition 4.5. Let the circle act on a connected compact $2 n$-dimensional symplectic manifold $(M, \omega)$ with moment map $\phi: M \rightarrow \mathbb{R}$. Assume $[\omega]$ is an integral class and $M^{S^{1}}=\left\{P_{0}, \cdots, P_{\frac{n}{2}-1}, P_{\frac{n}{2}}, P_{\left(\frac{n}{2}\right)^{\prime}}, P_{\frac{n}{2}+1} \cdots, P_{n}\right\}$. Assume the integer $\phi\left(P_{n-1}\right)-\phi\left(P_{0}\right)=\phi\left(P_{n}\right)-\phi\left(P_{1}\right)$ occurs as a weight of the $S^{1}$-action at some fixed point. Then the set of weights at any $P_{i}$ is

$$
\left\{w_{i j}=\phi\left(P_{j}\right)-\phi\left(P_{i}\right)\right\}_{j \neq i, n-i} \text {. }
$$

Moreover,

$$
\phi\left(P_{k}\right)-\phi\left(P_{0}\right)=\phi\left(P_{n}\right)-\phi\left(P_{n-k}\right), \text { for any } k \text { with } 1 \leq k \leq \frac{n}{2} .
$$

Hence the sets of weights at the fixed points are isomorphic to those of the standard circle action on $\widetilde{G}_{2}\left(\mathbb{R}^{n+2}\right)$, with $n \geq 2$ even, as in Example [2.1.

Proof. Lemma 3.3 gives the results for $n=2$. For $n>2$, Lemma 4.2 gives (4.6) for $P_{0}, P_{n-1}, P_{n}$ and $P_{1}$, and (4.7) for $k=1$. By Lemma 4.8 below, for any $i=0,1, n-1, n, w_{i j}$ is a weight between $P_{i}$ and $P_{j}$ for each $j \neq i, n-i$.

The rest of the proof is similar to that of Proposition 6.10 in [9], keeping in mind that $\phi\left(P_{n}\right)-\phi\left(P_{0}\right)$ is not a weight, by Proposition 3.6. We use induction. Fix $i$ with $2 \leq i \leq \frac{n}{2}$. Assume that for each $k$ with $0 \leq k<i$, (4.6) holds for $P_{k}$ and $P_{n-k}, w_{k j}$ is a weight between $P_{k}$ and $P_{j}$ for each $j \neq k, n-k$, and $w_{n-k, j}$ is a weight between $P_{n-k}$ and $P_{j}$ for each $j \neq n-k, k$, moreover, (4.7) holds for all $1 \leq k \leq i-1$. We need to prove all these hold if we replace $k$ by $i$. Proceed similarly as in the proof of Proposition 6.10 in [9], and use Lemma 4.8 below. 
Lemma 4.8 can be proved similarly as Lemmas 6.1 and 6.9 in [9].

Lemma 4.8. Let the circle act on a connected compact $2 n$-dimensional symplectic manifold $(M, \omega)$ with moment map $\phi: M \rightarrow \mathbb{R}$, where $n>2$ is even. Assume $M^{S^{1}}=\left\{P_{0}, \cdots, P_{\frac{n}{2}-1}, P_{\frac{n}{2}}, P_{\left(\frac{n}{2}\right)^{\prime}}, P_{\frac{n}{2}+1} \cdots, P_{n}\right\}$ and $[\omega]$ is an integral class. Assume for a fixed $i$ with $0 \leq i \leq \frac{n}{2}$, the set of weights at each $P_{k}$ with $k \leq i$ and $k \geq n-i$ is

$$
\left\{w_{k j}=\phi\left(P_{j}\right)-\phi\left(P_{k}\right)\right\}_{j \neq k, n-k},
$$

and for each $k<i$ and $k>n-i, w_{k j}$ is a weight between $P_{k}$ and $P_{j}$ for each $j \neq k, n-k$. Then $w_{i j}$ is a weight between $P_{i}$ and $P_{j}$ for each $j \neq i, n-i$, and $w_{n-i, j}$ is a weight between $P_{n-i}$ and $P_{j}$ for each $j \neq n-i, i$.

\section{FROM THE SETS OF WEIGHTS TO THE INTEGRAL COHOMOLOGY RING AND TOTAL CHERN CLASS OF $M$}

In this section, we use the sets of weights of the $S^{1}$-action to determine the integral cohomology ring and total Chern class of the manifold, proving (1) and (2) of Theorem 2

We will do this in a few steps.

Lemma 5.1. Let the circle act on a connected compact $2 n$-dimensional symplectic manifold $(M, \omega)$ with moment map $\phi: M \rightarrow \mathbb{R}$. Assume $[\omega]$ is a primitive integral class and $M^{S^{1}}=\left\{P_{0}, \cdots, P_{\frac{n}{2}-1}, P_{\frac{n}{2}}, P_{\left(\frac{n}{2}\right)^{\prime}}, P_{\frac{n}{2}+1} \cdots, P_{n}\right\}$. Let $x=[\omega]$. Then the following conditions are equivalent:

(1) There exist classes $y, z \in H^{n}(M ; \mathbb{Z})$ such that the generators of $H^{2 i}(M ; \mathbb{Z})$ with $0 \leq 2 i \leq n$ are $1, x, \cdots, x^{\frac{n}{2}-1}$, $y$ and $z$, where $y+z=x^{\frac{n}{2}}$.

(2) For any $i \in\left\{0,1, \cdots, \frac{n}{2},\left(\frac{n}{2}\right)^{\prime}\right\}$, the product $\Lambda_{i}^{-}$of the negative weights at $P_{i}$ is

$$
\Lambda_{i}^{-}=\prod_{\operatorname{index}\left(P_{j}\right)<\operatorname{index}\left(P_{i}\right)}\left(\phi\left(P_{j}\right)-\phi\left(P_{i}\right)\right) .
$$

(3) For any $i \in\left\{\frac{n}{2},\left(\frac{n}{2}\right)^{\prime}, \frac{n}{2}+1, \cdots, n\right\}$, the product $\Lambda_{i}^{+}$of the positive weights at $P_{i}$ is

$$
\Lambda_{i}^{+}=\prod_{\operatorname{index}\left(P_{j}\right)>\operatorname{index}\left(P_{i}\right)}\left(\phi\left(P_{j}\right)-\phi\left(P_{i}\right)\right) .
$$

Proof. Let us prove (1) and (2) are equivalent. Let $\left\{\widetilde{\alpha}_{i}\right\}_{i \in\left\{0, \cdots, \frac{n}{2},\left(\frac{n}{2}\right)^{\prime}, \cdots, n\right\}}$ be the basis of $H_{S^{1}}^{*}(M ; \mathbb{Z})$ as an $H^{*}\left(\mathbb{C} \mathbb{P}^{\infty} ; \mathbb{Z}\right)$-module as in Proposition 2.5. Let $\left\{\alpha_{i}\right\}_{i \in\left\{0, \cdots, \frac{n}{2},\left(\frac{n}{2}\right)^{\prime}, \cdots, n\right\}}$ be the restriction of the above basis to ordinary cohomology, which is a basis of the ordinary cohomology of $M$.

First, consider $0 \leq i<\frac{n}{2}$. Since $\left.\prod_{j<i}\left(\widetilde{u}+\phi\left(P_{j}\right) t\right)\right|_{P_{j}}=0$ for all $j<i$, where $\widetilde{u}$ is the class in Lemma 2.7, by Corollary 2.6.

$$
\prod_{j<i}\left(\widetilde{u}+\phi\left(P_{j}\right) t\right)=a_{i} \widetilde{\alpha}_{i}, \text { where } a_{i} \in \mathbb{Z} \text {. }
$$


Restricting (5.4) to ordinary cohomology, we get $[\omega]^{i}=a_{i} \alpha_{i}$. Restricting (5.4) to $P_{i}$, we get $\prod_{j<i}\left(\phi\left(P_{j}\right)-\phi\left(P_{i}\right)\right)=a_{i} \Lambda_{i}^{-}$. Hence $\alpha_{i}=[\omega]^{i}$ if and only if (5.2) holds for $i$ (with $0 \leq i<\frac{n}{2}$ ).

Similarly, by Corollary 2.6.

$$
\prod_{j<\frac{n}{2}}\left(\widetilde{u}+\phi\left(P_{j}\right) t\right)=b \widetilde{\alpha}_{\frac{n}{2}}+b^{\prime} \widetilde{\alpha}_{\left(\frac{n}{2}\right)^{\prime}}, \text { where } b, b^{\prime} \in \mathbb{Z} .
$$

Restricting (5.5) to ordinary cohomology, we get $[\omega]^{\frac{n}{2}}=b \alpha_{\frac{n}{2}}+b^{\prime} \alpha_{\left(\frac{n}{2}\right)^{\prime}}$. Restricting (5.5) respectively to $P_{\frac{n}{2}}$ and $P_{\left(\frac{n}{2}\right)^{\prime}}$, we get

$$
\prod_{j<\frac{n}{2}}\left(\phi\left(P_{j}\right)-\phi\left(P_{\frac{n}{2}}\right)\right)=b \Lambda_{\frac{n}{2}}^{-}, \text {and } \prod_{j<\frac{n}{2}}\left(\phi\left(P_{j}\right)-\phi\left(P_{\left(\frac{n}{2}\right)^{\prime}}\right)\right)=b^{\prime} \Lambda_{\left(\frac{n}{2}\right)^{\prime}}^{-}
$$

Hence $[\omega]^{\frac{n}{2}}=\alpha_{\frac{n}{2}}+\alpha_{\left(\frac{n}{2}\right)^{\prime}}$ if and only if (5.2) holds for $i=\frac{n}{2}$ and $\left(\frac{n}{2}\right)^{\prime}$. We take $y=\alpha_{\frac{n}{2}}$ and $z=\alpha_{\left(\frac{n}{2}\right)^{\prime}}$.

Using $-\phi$, we can get the Poincaré dual classes $y^{\prime}$ and $z^{\prime}$ of the above $y$ and $z$. The existence of the new basis $1, x, \cdots, x^{\frac{n}{2}-1}, y^{\prime}, z^{\prime}$ is equivalent to (1), and by the same argument as above is equivalent to (3).

Next, we aim to prove Proposition 5.10. As a preparation, we first prove two lemmas.

Lemma 5.6. Let the circle act on a connected compact $2 n$-dimensional symplectic manifold $(M, \omega)$ with moment map $\phi: M \rightarrow \mathbb{R}$. Assume

$$
M^{S^{1}}=\left\{P_{0}, \cdots, P_{\frac{n}{2}-1}, P_{\frac{n}{2}}, P_{\left(\frac{n}{2}\right)^{\prime}}, P_{\frac{n}{2}+1} \cdots, P_{n}\right\} .
$$

Then for any $i \in\left\{\left(\frac{n}{2}\right)^{\prime}, \frac{n}{2}+1, \cdots, n-1\right\}$,

$$
\left.\widetilde{\alpha}_{i}\right|_{P_{i+1}}=-\frac{\Lambda_{i+1}}{\Lambda_{i}^{+}} \prod_{j>i+1} \frac{\phi\left(P_{j}\right)-\phi\left(P_{i}\right)}{\phi\left(P_{j}\right)-\phi\left(P_{i+1}\right)} t^{i} .
$$

Here, the $\widetilde{\alpha}_{i}$ 's are the classes in Proposition 2.5, $\Lambda_{i+1}$ is the product of all the weights at $P_{i+1}$, and we use the convention $\left(\frac{n}{2}\right)^{\prime}+1=\frac{n}{2}+1$ and $t^{\left(\frac{n}{2}\right)^{\prime}}=t^{\frac{n}{2}}$.

Proof. For any $i \in\left\{\left(\frac{n}{2}\right)^{\prime}, \frac{n}{2}+1, \cdots, n-1\right\}$, note that

$$
\operatorname{deg}\left(\widetilde{\alpha}_{i} \cdot \prod_{j>i+1}\left(\widetilde{u}+\phi\left(P_{j}\right) t\right)\right)<2 n .
$$

Using Theorem 3 to integrate this class on $M$, we get

$$
0=\frac{\left.\widetilde{\alpha}_{i}\right|_{P_{i}} \cdot \prod_{j>i+1}\left(\phi\left(P_{j}\right)-\phi\left(P_{i}\right)\right)}{\Lambda_{i}}+\frac{\left.\widetilde{\alpha}_{i}\right|_{P_{i+1}} \cdot \prod_{j>i+1}\left(\phi\left(P_{j}\right)-\phi\left(P_{i+1}\right)\right)}{\Lambda_{i+1}} .
$$

Solving this, we get (5.7).

Lemma 5.8. Let the circle act on a connected compact $2 n$-dimensional symplectic manifold $(M, \omega)$ with moment map $\phi: M \rightarrow \mathbb{R}$. Assume $[\omega]$ is a 
primitive integral class and $M^{S^{1}}=\left\{P_{0}, \cdots, P_{\frac{n}{2}-1}, P_{\frac{n}{2}}, P_{\left(\frac{n}{2}\right)^{\prime}}, P_{\frac{n}{2}+1} \cdots, P_{n}\right\}$. Then for any $i \in\left\{\left(\frac{n}{2}\right)^{\prime}, \frac{n}{2}+1, \cdots, n-1\right\},[\omega] \alpha_{i}=\alpha_{i+1}$ if and only if

$$
\left.\widetilde{\alpha}_{i}\right|_{P_{i+1}}=\frac{\Lambda_{i+1}^{-}}{\phi\left(P_{i}\right)-\phi\left(P_{i+1}\right)} t^{i} .
$$

Here, the $\widetilde{\alpha}_{i}$ 's and $\alpha_{i}$ 's are the classes in Proposition 2.5, and we use the convention $\left(\frac{n}{2}\right)^{\prime}+1=\frac{n}{2}+1$ and $t^{\left(\frac{n}{2}\right)^{\prime}}=t^{\frac{n}{2}}$.

Proof. For any $i \in\left\{\left(\frac{n}{2}\right)^{\prime}, \frac{n}{2}+1, \cdots, n-1\right\}$, note that

$$
\begin{gathered}
\operatorname{deg}\left(\left(\widetilde{u}+\phi\left(P_{i}\right) t\right) \widetilde{\alpha}_{i}\right)=\operatorname{deg}\left(\widetilde{\alpha}_{i+1}\right), \text { and } \\
\left.\left(\left(\widetilde{u}+\phi\left(P_{i}\right) t\right) \widetilde{\alpha}_{i}\right)\right|_{P_{j}}=0, \quad \forall P_{j} \text { with } \phi\left(P_{j}\right)<\phi\left(P_{i+1}\right) .
\end{gathered}
$$

By Corollary 2.6, $\left(\widetilde{u}+\phi\left(P_{i}\right) t\right) \widetilde{\alpha}_{i}=a_{i+1} \widetilde{\alpha}_{i+1}$ with $a_{i+1} \in \mathbb{Z}$. Restricting it respectively to ordinary cohomology and to $P_{i+1}$, we obtain the claim.

Proposition 5.10. Let the circle act on a connected compact $2 n$-dimensional symplectic manifold $(M, \omega)$ with moment map $\phi: M \rightarrow \mathbb{R}$. Assume $[\omega]$ is a primitive integral class and $M^{S^{1}}=\left\{P_{0}, \cdots, P_{\frac{n}{2}-1}, P_{\frac{n}{2}}, P_{\left(\frac{n}{2}\right)^{\prime}}, P_{\frac{n}{2}+1} \cdots, P_{n}\right\}$. Let $x=[\omega]$. Then the following conditions are equivalent:

(1) There exist classes $y, z \in H^{n}(M ; \mathbb{Z})$ such that the generators of $H^{*}(M ; \mathbb{Z})$ are $1, x, \cdots, x^{\frac{n}{2}-1}, y, z, x y=x z=\frac{1}{2} x^{\frac{n}{2}+1}, x^{2} y=$ $x^{2} z=\frac{1}{2} x^{\frac{n}{2}+2}, \cdots, x^{\frac{n}{2}} y=x^{\frac{n}{2}} z=\frac{1}{2} x^{n}$, where $x^{\frac{n}{2}}=y+z$.

(2) Lemma 5.1 (2) holds, and for any $i$ with $\frac{n}{2}+1 \leq i \leq n$, the product of the negative weights at $P_{i}$ is

$$
\Lambda_{i}^{-}=\frac{\prod_{\text {index }\left(P_{j}\right)<\operatorname{index}\left(P_{i}\right)}\left(\phi\left(P_{j}\right)-\phi\left(P_{i}\right)\right)}{\left(\phi\left(P_{\frac{n}{2}}\right)-\phi\left(P_{i}\right)\right)+\left(\phi\left(P_{\left(\frac{n}{2}\right)^{\prime}}\right)-\phi\left(P_{i}\right)\right)} .
$$

(3) Lemma 5.1 (3) holds, and for any $i$ with $0 \leq i \leq \frac{n}{2}-1$, the product of the positive weights at $P_{i}$ is

$$
\Lambda_{i}^{+}=\frac{\prod_{\text {index }\left(P_{j}\right)>\operatorname{index}\left(P_{i}\right)}\left(\phi\left(P_{j}\right)-\phi\left(P_{i}\right)\right)}{\left(\phi\left(P_{\frac{n}{2}}\right)-\phi\left(P_{i}\right)\right)+\left(\phi\left(P_{\left(\frac{n}{2}\right)^{\prime}}\right)-\phi\left(P_{i}\right)\right)} .
$$

Proof. Let $\left\{\widetilde{\alpha}_{i}\right\}_{i \in\left\{0, \cdots, \frac{n}{2},\left(\frac{n}{2}\right)^{\prime}, \cdots, n\right\}}$ and $\left\{\alpha_{i}\right\}_{i \in\left\{0, \cdots, \frac{n}{2},\left(\frac{n}{2}\right)^{\prime}, \cdots, n\right\}}$ be respectively the basis of the equivariant and ordinary cohomology of $M$ as in Proposition 2.5.

First, consider $i=\frac{n}{2}+1$. Since $\operatorname{deg}\left(\prod_{j \leq \frac{n}{2}}\left(\widetilde{u}+\phi\left(P_{j}\right) t\right)\right)=n+2$, and $\left.\prod_{j \leq \frac{n}{2}}\left(\widetilde{u}+\phi\left(P_{j}\right) t\right)\right|_{P_{j}}=0$ for all $j \leq \frac{n}{2}$, by Corollary 2.6.

$$
\prod_{j \leq \frac{n}{2}}\left(\widetilde{u}+\phi\left(P_{j}\right) t\right)=a_{\left(\frac{n}{2}\right)^{\prime}} t \widetilde{\alpha}_{\left(\frac{n}{2}\right)^{\prime}}+b_{\frac{n}{2}+1} \widetilde{\alpha}_{\frac{n}{2}+1},
$$

where $a_{\left(\frac{n}{2}\right)^{\prime}}, b_{\frac{n}{2}+1} \in \mathbb{Z}$. Restricting (5.13) to ordinary cohomology, we get

$$
[\omega]^{\frac{n}{2}+1}=b_{\frac{n}{2}+1} \alpha \frac{n}{2}+1 .
$$


Restricting (5.13) to $P_{\left(\frac{n}{2}\right)^{\prime}}$, we get

$$
\prod_{j \leq \frac{n}{2}}\left(\phi\left(P_{j}\right)-\phi\left(P_{\left(\frac{n}{2}\right)^{\prime}}\right)\right)=a_{\left(\frac{n}{2}\right)^{\prime}} \Lambda_{\left(\frac{n}{2}\right)^{\prime}}^{-} .
$$

Restricting (5.13) to $P_{\frac{n}{2}+1}$, we get

$$
\prod_{j \leq \frac{n}{2}}\left(\phi\left(P_{j}\right)-\phi\left(P_{\frac{n}{2}+1}\right)\right)=\left.a_{\left(\frac{n}{2}\right)^{\prime}} \widetilde{\alpha}_{\left(\frac{n}{2}\right)^{\prime}}\right|_{P_{\frac{n}{2}+1}}+b_{\frac{n}{2}+1} \Lambda_{\frac{n}{2}+1}^{-} .
$$

Similarly, for any $i \geq \frac{n}{2}+2$, we can write

$$
\prod_{\frac{1}{2} \operatorname{index}\left(P_{j}\right)<i-1}\left(\widetilde{u}+\phi\left(P_{j}\right) t\right)=a_{i-1} t \widetilde{\alpha}_{i-1}+b_{i} \widetilde{\alpha}_{i},
$$

where $a_{i-1}, b_{i} \in \mathbb{Z}$. Restricting (5.14) to ordinary cohomology, we get

$$
[\omega]^{i}=b_{i} \alpha_{i} .
$$

Restricting (5.14) to $P_{i-1}$, we get

$$
\prod_{\frac{1}{2} \operatorname{index}\left(P_{j}\right)<i-1}\left(\phi\left(P_{j}\right)-\phi\left(P_{i-1}\right)\right)=a_{i-1} \Lambda_{i-1}^{-} .
$$

Restricting (5.14) to $P_{i}$, we get

$$
\prod_{\frac{1}{2} \operatorname{index}\left(P_{j}\right)<i-1}\left(\phi\left(P_{j}\right)-\phi\left(P_{i}\right)\right)=\left.a_{i-1} \widetilde{\alpha}_{i-1}\right|_{P_{i}}+b_{i} \Lambda_{i}^{-} .
$$

Let $y=\alpha_{\frac{n}{2}}$ and $z=\alpha_{\left(\frac{n}{2}\right)^{\prime}}$. We argue below that (1) and (2) are equivalent. Assume (1) holds. Then Lemma 5.1(2) holds, $b_{i}=2$ for all $\frac{n}{2}+1 \leq i \leq n$, and (5.9) holds. Using (5.2), we can get $a_{\left(\frac{n}{2}\right)^{\prime}}$, then get $\Lambda_{\frac{n}{2}+1}^{-}$. Inductively, we can get $a_{i-1}$, and then get $\Lambda_{i}^{-}$for all $i \geq \frac{n}{2}+2$.

Conversely, assume (2) holds. Then by Lemma 5.1, we have the generators of $H^{2 i}(M ; \mathbb{Z})$ for $0 \leq 2 i \leq n$ as in (1) and $x^{\frac{n}{2}}=y+z$, and Lemma 5.1 (3) holds. By (5.7), we get that (5.9) holds. Then Lemma 5.8 implies that $x^{k} z=\alpha_{\frac{n}{2}+k}$ for all $1 \leq k \leq \frac{n}{2}$. Moreover, from the above equalities, we can solve $a_{\left(\frac{n}{2}\right)^{\prime}}$ and all $a_{i-1}$, and then get $b_{i}=2$ for all $\frac{n}{2}+1 \leq i \leq n$. Note that $x^{k} y+x^{k} z=x^{\frac{n}{2}+k}$ for all $1 \leq k \leq \frac{n}{2}$. Hence $x^{k} y=x^{k} z=\frac{1}{2} x^{\frac{n}{2}+k}=\alpha_{\frac{n}{2}+k}$ are generators of $H^{n+2 k}(M ; \mathbb{Z})$ for all $1 \leq k \leq \frac{n}{2}$.

Using $-\phi$ as a Morse function, we get the Poincaré dual of the classes in (1), they form a new basis (satisfying the same relations). The existence of the new basis is equivalent to (1), and by the same arguments as above, is equivalent to $(3)$.

Next, having Propositions 4.5 and 5.10, we look at the ring structure of the manifold. 
Proposition 5.15. Let the circle act on a connected compact $2 n$-dimensional symplectic manifold $(M, \omega)$ with moment map $\phi: M \rightarrow \mathbb{R}$. Assume $[\omega]$ is an integral class and $M^{S^{1}}=\left\{P_{0}, \cdots, P_{\frac{n}{2}-1}, P_{\frac{n}{2}}, P_{\left(\frac{n}{2}\right)^{\prime}}, P_{\frac{n}{2}+1} \cdots, P_{n}\right\}$. Assume the integer $\phi\left(P_{n-1}\right)-\phi\left(P_{0}\right)=\phi\left(P_{n}\right)-\phi\left(P_{1}\right)$ occurs as a weight of the $S^{1}$-action at some fixed point. Then Proposition 5.10 (1) holds, moreover,

$$
\begin{gathered}
x^{\frac{n}{2}} y=x^{\frac{n}{2}} z=y z, y^{2}=z^{2}=0, \text { when } \operatorname{dim}(M)=4 m \text { with } m \text { odd, and } \\
x^{\frac{n}{2}} y=x^{\frac{n}{2}} z=y^{2}=z^{2}, y z=0, \text { when } \operatorname{dim}(M)=4 m \text { with } m \text { even. }
\end{gathered}
$$

Hence, as rings, $H^{*}(M ; \mathbb{Z}) \cong H^{*}\left(\widetilde{G}_{2}\left(\mathbb{R}^{n+2}\right) ; \mathbb{Z}\right)$ with $n \geq 2$ even.

Proof. By Proposition 4.5, Proposition 5.10 (2) (and (3)) holds. Hence Proposition 5.10 (1) holds. It remains to determine the relations of $y^{2}, z^{2}$ and $y z$ with the top degree generators. First, since $x^{\frac{n}{2}}=y+z$, we have $x^{\frac{n}{2}} y=y^{2}+y z$ and $x^{\frac{n}{2}} z=z^{2}+y z$. Since $x^{\frac{n}{2}} y=x^{\frac{n}{2}} z$, we have $y^{2}=z^{2}$.

Recall that $y=\alpha_{\frac{n}{2}}$ and $z=\alpha_{\left(\frac{n}{2}\right)^{\prime}}$, where $\alpha_{\frac{n}{2}}$ and $\alpha_{\left(\frac{n}{2}\right)^{\prime}}$ are respectively the restrictions of $\widetilde{\alpha}_{\frac{n}{2}}$ and $\widetilde{\alpha}_{\left(\frac{n}{2}\right)^{\prime}}$ to ordinary cohomology. For any $k \geq \frac{n}{2}+1$, note that

$$
\left.\left(\widetilde{\alpha}_{\frac{n}{2}} \cdot \prod_{j \in\left\{\frac{n}{2}, \frac{n}{2}+1, \cdots, k-1\right\}}\left(\widetilde{u}+\phi\left(P_{j}\right) t\right)\right)\right|_{P_{j}}=0, \forall P_{j} \text { with } \phi\left(P_{j}\right)<\phi\left(P_{k}\right) .
$$

By Corollary 2.6,

$$
\widetilde{\alpha}_{\frac{n}{2}} \cdot \prod_{j \in\left\{\frac{n}{2}, \frac{n}{2}+1, \cdots, k-1\right\}}\left(\widetilde{u}+\phi\left(P_{j}\right) t\right)=a_{k} \widetilde{\alpha}_{k}, \text { with } a_{k} \in \mathbb{Z} .
$$

By restricting it to ordinary cohomology, we see that $a_{k}=1$. Then restricting it to $P_{k}$, we get

$$
\left.\widetilde{\alpha}_{\frac{n}{2}}\right|_{P_{k}}=\frac{\Lambda_{k}^{-}}{\prod_{j \in\left\{\frac{n}{2}, \frac{n}{2}+1, \cdots, k-1\right\}}\left(\phi\left(P_{j}\right)-\phi\left(P_{k}\right)\right)} .
$$

Similarly, we have

$$
\left.\widetilde{\alpha}_{\left(\frac{n}{2}\right)^{\prime}}\right|_{P_{k}}=\frac{\Lambda_{k}^{-}}{\prod_{j \in\left\{\left(\frac{n}{2}\right)^{\prime}, \frac{n}{2}+1, \cdots, k-1\right\}}\left(\phi\left(P_{j}\right)-\phi\left(P_{k}\right)\right)}, \quad \forall k \geq \frac{n}{2}+1 .
$$

By Theorem 3 ,

$$
\begin{gathered}
\int_{M} y^{2}=\int_{M}\left(\widetilde{\alpha}_{\frac{n}{2}}\right)^{2}=\frac{\Lambda_{\frac{n}{2}}^{-} \Lambda_{\frac{n}{2}}^{-}}{\Lambda_{\frac{n}{2}}}+\sum_{k \geq \frac{n}{2}+1} \frac{\left.\left(\widetilde{\alpha}_{\frac{n}{2}}\right)^{2}\right|_{P_{k}}}{\Lambda_{k}}=\frac{\Lambda_{\frac{n}{2}}^{-}}{\Lambda_{\frac{n}{2}}^{+}}+\sum_{k \geq \frac{n}{2}+1} \frac{\left.\left(\widetilde{\alpha}_{\frac{n}{2}}\right)^{2}\right|_{P_{k}}}{\Lambda_{k}} \text {, and } \\
\int_{M} y z=\int_{M} \widetilde{\alpha}_{\frac{n}{2}} \widetilde{\alpha}_{\left(\frac{n}{2}\right)^{\prime}}=\sum_{k \geq \frac{n}{2}+1} \frac{\left.\left.\widetilde{\alpha}_{\frac{n}{2}}\right|_{P_{k}} \cdot \widetilde{\alpha}_{\left(\frac{n}{2}\right)^{\prime}}\right|_{P_{k}}}{\Lambda_{k}} .
\end{gathered}
$$

Since the integrals $\int_{M} y^{2}$ and $\int_{M} y z$ should not depend on the moment map values of the fixed points, we may assume that $\phi\left(P_{\frac{n}{2}}\right)=\phi\left(P_{\left(\frac{n}{2}\right)^{\prime}}\right)$. 
Then by the expressions above, $\left.\widetilde{\alpha}_{\frac{n}{2}}\right|_{P_{k}}=\left.\widetilde{\alpha}_{\left(\frac{n}{2}\right)^{\prime}}\right|_{P_{k}}, \forall k \geq \frac{n}{2}+1$, and by Proposition 4.5, $\Lambda_{\frac{n}{2}}^{-}=(-1)^{\frac{n}{2}} \Lambda_{\frac{n}{2}}^{+}$. These together give

$$
\int_{M} y^{2}-\int_{M} y z=(-1)^{\frac{n}{2}}
$$

While $x^{\frac{n}{2}} y=y^{2}+y z$ gives

$$
\int_{M} y^{2}+\int_{M} y z=\int_{M} x^{\frac{n}{2}} y=1
$$

Hence

$$
\int_{M} y^{2}=\frac{1+(-1)^{\frac{n}{2}}}{2}
$$

Finally, we use the weights in Proposition 4.5 to determine $c(M)$.

Proposition 5.16. Let the circle act on a connected compact $2 n$-dimensional symplectic manifold $(M, \omega)$ with moment $\operatorname{map} \phi: M \rightarrow \mathbb{R}$. Assume $[\omega]$ is an integral class and $M^{S^{1}}=\left\{P_{0}, \cdots, P_{\frac{n}{2}-1}, P_{\frac{n}{2}}, P_{\left(\frac{n}{2}\right)^{\prime}}, P_{\frac{n}{2}+1}, \cdots, P_{n}\right\}$. Assume $\phi\left(P_{n-1}\right)-\phi\left(P_{0}\right)=\phi\left(P_{n}\right)-\phi\left(P_{1}\right)$ holds and this integer occurs as a weight of the $S^{1}$-action at some fixed point. Then the total Chern class of $M$ is $c(M)=\frac{(1+[\omega])^{n+2}}{1+2[\omega]}$, hence is isomorphic to $c\left(\widetilde{G}_{2}\left(\mathbb{R}^{n+2}\right)\right)$.

Proof. By the injectivity theorem $([6,14])$, the restriction map

$$
H_{S^{1}}^{*}(M ; \mathbb{Z}) \rightarrow H_{S^{1}}^{*}\left(M^{S^{1}} ; \mathbb{Z}\right)
$$

is injective. Let $I=\left\{0, \cdots, \frac{n}{2},\left(\frac{n}{2}\right)^{\prime}, \cdots, n\right\}$. Consider the class

$$
\alpha=\frac{\prod_{j \in I}\left(1+\widetilde{u}+\phi\left(P_{j}\right) t\right)}{1+\left(\widetilde{u}+\phi\left(P_{0}\right) t\right)+\left(\widetilde{u}+\phi\left(P_{n}\right) t\right)} .
$$

By Proposition 4.5, $\left(\phi\left(P_{0}\right)-\phi\left(P_{i}\right)\right)+\left(\phi\left(P_{n}\right)-\phi\left(P_{i}\right)\right)=\phi\left(P_{n-i}\right)-\phi\left(P_{i}\right)$ for all $i \in I$. Using this, and Proposition 4.5, we can check that

$$
\left.\alpha\right|_{P_{i}}=\left.c^{S^{1}}(M)\right|_{P_{i}}, \quad \forall i \in I .
$$

Hence $c^{S^{1}}(M)=\alpha$. Restricting this to ordinary cohomology, we obtain our claim.

\section{REFERENCES}

[1] M. Atiyah and R. Bott, The moment map and equivariant cohomology, Topology, 23 (1984), 1-28.

[2] N. Berline and M. Vergne, Classes caractéristiques équivariantes, formule de localisation en cohomologie équivariante, C. R. Acad. Sci. Paris, 295 (1982), 539-541.

[3] L. Godinho and S. Sabatini, New tools for classifying Hamiltonian circle actions with isolated fixed points, Foundations in Computational Mathematics, 14(2014), 791-860.

[4] A. Hattori, $S^{1}$ actions on unitary manifolds and quasi-ample line bundles, J. Fac. Sci. Univ. Tokyo Sect. IA, Math., 31, no. 3 (1984), 433-486. 
[5] D. Jang and S. Tolman, Hamiltonian circle actions on eight dimensional manifolds with minimal fixed sets, Transformation groups, vol. 22, no. 2, 2017, 353-359.

[6] F. Kirwan, Cohomology of Quotients in Symplectic and Algebraic Geometry, Princeton University Press, 1984.

[7] H. Li, Certain circle actions on Kähler manifolds, International Mathematics Research Notices, Vol. 2014, No. 18, 5187-5202.

[8] H. Li, Hamiltonian circle actions with fixed point set almost minimal, Math. Z., 293, (2019), no. 3, 1315-1336.

[9] H. Li, Hamiltonian circle actions with minimal isolated fixed points, arXiv: $1407.1948 \mathrm{v} 5$.

[10] H. Li, M. Olbermann and D. Stanley, One connectivity and finiteness of Hamiltonian $S^{1}$-manifolds with minimal fixed sets, J. of Lond. Math. Soc. (2) 92 (2015), no. 2, 284-310.

[11] H. Li and S. Tolman, Hamiltonian circle actions with minimal fixed sets, International Journal of Mathematics 23, no. 8 (2012), 1250071.

[12] D. McDuff, Some 6-dimensional Hamiltonian $S^{1}$ manifolds, J. of Topology, 2 (2009), no. 3, 589-623.

[13] S. Tolman, On a symplectic generalization of Petrie's conjecture, Trans. Amer. Math. Soc., 362 (2010), 3963-3996.

[14] S. Toman and J. Weitsman, The cohomology rings of symplectic quotients, Comm. Anal. Geom., 11 (2003), no. 4, 751-773.

School of Mathematical Sciences, Soochow University, Suzhou, 215006, China

Email address: hui.li@suda.edu.cn 